\title{
Distributed Subchannel Assignment in an OFDMA Relay
}

\author{
Ari Hottinen \\ Radio Communications CTC \\ Nokia Research Center \\ P.O. Box 407, FIN-00045 Nokia Group, Finland \\ ari.hottinen@nokia.com
}

\author{
Tiina Heikkinen \\ Department of Computer Science \\ P.O. Box 68, FIN-00014, University of Helsinki, \\ Finland \\ tiina.heikkinen@cs.helsinki.fi
}

\begin{abstract}
The paper studies a distributed OFDMA system in which the transmitting nodes (source and relay) have access to channel information and to some interference-related information. Distributed subchannel allocation is modelled as a noncooperative game where the players are the source nodes. Channel-aware source nodes opportunistically select their subchannels while a channel-aware relay node assigns the input subchannels at relay to appropriate output subchannels in a way that maximizes the sum of utilities in terms of signal-to-noise ratio. Numerical examples illustrate the performance of distributed subchannel assignment; opportunistic channel allocation implies a trade-off between the average capacity and fairness.
\end{abstract}

\section{Keywords}

Relay network, resource allocation, noncooperative game theory, OFDM

\section{INTRODUCTION}

Relays have been applied recently in diverse applications in wireless systems, including large-scale broadcast networks such DVB-T and DVB-H and their use has been proposed recently e.g. in IEEE 802.16 standardization for OFDMA systems [13]. Relay nodes are traditionally used to improve communication between a source and a destination by decomposing one source-to-destination link into one or multiple shorter links.

In this paper, we address a subchannel selection problem in a multi-channel relay involving multiple source nodes, one relay node and one destination node. The signal that is transmitted by a given source and the relay, respectively, comprises several subchannels. For example, one or some subset of available subcarriers of an OFDM system form one subchannel. The relay receives these subchannels and may reassign a received input subchannel to a different output subchannel, which is then transmitted to another relay node or to a destination node.

Permission to make digital or hard copies of all or part of this work for personal or classroom use is granted without fee provided that copies are not made or distributed for profit or commercial advantage and that copies bear this notice and the full citation on the first page. To copy otherwise, to republish, to post on servers or to redistribute to lists, requires prior specific permission and/or a fee.

Valuetools '07, October 23-25, 2007, Nantes, France

Copyright 2007 ICST 978-963-9799-00-4.
A heuristic algorithm for determining the subchannel reassignment in the case of a single source node was proposed by the authors in [7], and independently in [5] for MIMOOFDM systems. In this paper, we extend [7] to the case of multiple source nodes that compete for a limited number of subchannels. With a large number of users the size (complexity) of the optimal subchannel allocation problem can be very large. This motivates the study of distributed models and algorithms. Noncooperative game theory [3] is applicable to study distributed resource allocation. In a game theoretic model of distributed resource allocation, channelaware nodes are assumed to assign the subchannels in a way that maximizes individual utilities (e.g. in terms of signalto-noise ratio). Assuming noncooperative resource allocation the source nodes only need local channel information to decide the individually optimal channel allocation.

Recent work [6] suggests that distributed resource allocation in a congested network can be modelled as a potential game where the potential refers to an implicit joint objective function of the distributed system. The subchannel allocation game studied in this paper is another example of a potential game (subchannel allocation was not considered in [6]). In a potential game, greedy best-reply dynamics will converge to a Nash equilibrium.

The paper is structured as follows. Section 2 recalls the OFDM signal model for a two-hop relaying scheme. Section 3 discusses the subchannel assignment problem, assuming orthogonal subchannels. A game model is presented for distributed subchannel allocation. The subchannel allocation problem is also formulated as a centralized problem. For simplicity, transmit power is first assumed to be constant. Section 4 extends the subchannel allocation model to transmit power allocation. Numerical examples of distributed subchannel allocation are discussed in section 5 . Section 6 concludes.

\section{SIGNAL MODEL}

\section{Single Source Node}

Consider first the subchannel allocation problem in a representative two-hop relay link with a given subset of orthogonal subchannels. In such a system, assume that a network that has a single source node (Node 1 ) a relay node (Node 2) and a destination node (Node 3), as depicted in Fig. 1.

We consider a system with $P$ subcarriers and let $\mathbf{F}$ denote a $P \times P$ inverse DFT (IDFT) matrix, where $[\mathbf{F}]_{p, q}=$ $1 / \sqrt{P} \exp (j 2 \pi(p-1)(q-1) / P)$. The DFT matrix applied at an OFDM receiver is given by $\mathbf{F}^{\dagger}$, the transpose conjugate 
of $\mathbf{F}$. We assume that the signal is transmitted through a finite impulse response (FIR) channel of length $L$ and that a cyclic prefix of length $L_{c}>L$ is used at the transmitter. We have a source node (Node 1 ), relay node (Node 2), and a destination node (Node 3). The circulant convolution matrix $\mathbf{H}$, obtained after removing the cyclic prefix at receiver has entries $[\mathbf{H}]_{p, q}=h((p-q) \bmod P)$, where $h(l)$ designates the $l$ th channel tap. Using this notation, $\mathbf{H}_{12}$ denotes the circulant channel between the source and the relay, and correspondingly, $\mathbf{H}_{23}$ the channel between the relay and destination. The effective channel between nodes $k$ and $l$ is

$$
\mathbf{D}_{k l}=\mathbf{F H}_{k l} \mathbf{F}^{\dagger}=\operatorname{diag}\left(d_{k l}(0), \ldots, d_{k l}(P-1)\right),
$$

with $d_{k l}(p)=\sum_{l=1}^{L} h_{k l}(l) \exp (-\mathrm{j} 2 \pi l p / P)$.

The source transmits $\mathbf{F}^{\dagger} \mathbf{x}$, where $\mathbf{x}$ is the symbol vector. The relay node applies DFT by multiplying the received signal with $\mathbf{F}$, and possibly permutes the subchannels using a permutation matrix $\mathbf{T}$. The permuted signal is transmitted to the destination in a similar fashion using amplify and forward (AF) protocol. Using equation (1) the received signal at destination is

$$
\mathbf{y}=\mathbf{D}_{23} \mathbf{T} \Lambda\left(\mathbf{D}_{12} \mathbf{x}+\mathbf{n}_{2}\right)+\mathbf{n}_{3},
$$

where $\mathbf{n}_{k}$ is the noise at the receiver of Node $k$ and $\Lambda$ is a diagonal matrix for which the $p$ th diagonal element assumes value

$$
\lambda[p]=\left(\left|d_{12}[p]\right|^{2}+\sigma_{2}[p]^{2}\right)^{-1 / 2},
$$

thereby normalizing the transmit power to unity. A extension of the model to transmit power control is presented in section 4 . The $p$ th element of noise vector, $n_{k}[p]$, affecting the $p$ th subcarrier at Node $k$, is modelled as a zero mean Gaussian random variable with power $\sigma_{k}[p]^{2}$. A signal that is transmitted from the source node using subcarrier $p^{\prime}$ is received at the $p$ th subcarrier at the destination Node 3

$$
y[p]=d_{23}[p] \lambda[p]\left(d_{12}\left[p^{\prime}\right] x+n_{2}\left[p^{\prime}\right]\right)+n_{3}[p],
$$

where $d_{k l}[p]$ is the effective complex channel between Node $k$ and Node $l$ for subcarrier $p$, and $n_{k}$ is the noise at the receiver of Node $k$. In the permutation matrix in equation (2) the element $t_{p, p^{\prime}}=1$ if received subcarrier $p^{\prime}$ is assigned to subcarrier $p$ at relay output, otherwise $t_{p, p^{\prime}}=0$.

For a given assignment $\left(p, p^{\prime}\right)$ the received signal power may be easily computed to be

$$
\mathrm{S}\left[p, p^{\prime}\right]=\left|d_{12}\left[p^{\prime}\right] \lambda[p] d_{23}[p]\right|^{2} .
$$

Likewise, the noise power is

$$
\mathrm{N}\left[p, p^{\prime}\right]=\sigma_{3}[p]^{2}+\left|\lambda[p] d_{23}[p]\right|^{2} \sigma_{2}\left[p^{\prime}\right]^{2}
$$

assuming that an amplify-and-forward relay is used. Consequently, the effective signal-to-noise ratio (SNR) at Node 3 for the assignment $\left(p, p^{\prime}\right)$ is

$$
\gamma_{3}\left[p, p^{\prime}\right]=\mathrm{S}\left[p, p^{\prime}\right] / \mathrm{N}\left[p, p^{\prime}\right] .
$$

The link-specific signal-to-noise-ratio in the $p^{\prime}$ th subcarrier for the source-relay link, and for the $p$ th subcarrier of the relay-destination link, are defined as

$$
\gamma_{12}\left[p^{\prime}\right]=\left|d_{12}\left[p^{\prime}\right]\right|^{2} / \sigma_{2}\left[p^{\prime}\right]^{2}
$$

and

$$
\gamma_{23}[p]=\left|d_{23}[p]\right|^{2} / \sigma_{3}[p]^{2}
$$

SOURCE

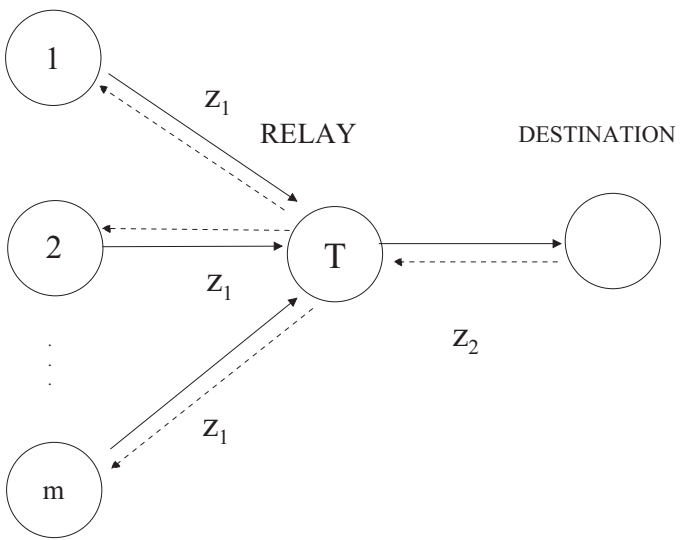

Figure 1: Relaying network with $m$ source nodes, one relay node and one destination node. Feedback messages between relay and each source, and destination and relay, are denoted by $z_{1}$ and $z_{2}$, respectively.

Using these notations, we can rewrite SNR at the destination node as

$$
\gamma_{3}\left[p, p^{\prime}\right]=\frac{\gamma_{12}\left[p^{\prime}\right] \gamma_{23}[p]}{\gamma_{12}\left[p^{\prime}\right]+\gamma_{23}[p]+1} .
$$

\section{Multiple Source Nodes}

In a multiuser system there are $m$ source nodes, each accessing the destination via the same relay. In the considered OFDMA case, the same subcarrier can be assigned only to one user at a time. To account for this, and aid distributed assignment, the relay node signals the source nodes vector $\mathbf{z}_{1} \in\{0,1\}^{P}$, which contains crude information pertaining to interference power in different subcarriers at the receiver of the relay node. In the simplest case, the relay sets $\mathbf{z}_{1}\left[p^{\prime}\right]=1$ if subcarrier $p^{\prime}$ is feasible for the considered source node, otherwise $\mathbf{z}_{1}\left[p^{\prime}\right]=0$. The feasibility can be determined by comparing the $\gamma_{3}\left[p^{\prime}\right]$ at destination to some threshold or simply by setting $\mathbf{z}_{1}\left[p^{\prime}\right]=0$ if subcarrier $p^{\prime}$ is assigned to another user. Analogously, the destination node signals the relay node $\mathbf{z}_{2} \in\{0,1\}^{P}$, as shown in Fig. 1. This is a crude form of signalling congestion-related information in the spirit of [6]. The interference information $\mathbf{z}_{1}$ and $\mathbf{z}_{2}$ is used at the relay node to modify the effective noise power in model (5) with

$$
\sigma_{2}^{2}\left[p^{\prime}\right] \leftarrow\left(1-\mathbf{z}_{1}\left[p^{\prime}\right]\right) \sigma_{0}^{2}+\mathbf{z}_{1}\left[p^{\prime}\right] \sigma_{2}^{2}\left[p^{\prime}\right]
$$

and

$$
\sigma_{3}^{2}[p] \leftarrow\left(1-\mathbf{z}_{2}[p]\right) \sigma_{0}^{2}+\mathbf{z}_{2}[p] \sigma_{3}^{2}[p],
$$

where we set $\sigma_{0}=\infty$ if a subchannel is punctured or unavailable for the user of interest.

\section{DISTRIBUTED SUBCHANNEL ALLOCA- TION}

Assume now there are $m$ source nodes competing for a limited number of subchannels. At the relay-destination link, 
the relay is assumed to choose an optimal subchannel allocation over users given channel information; let $\tau_{1}^{i}[p]=1$ if $i$ gets subchannel $p$ and let $\tau_{1}^{i}[p]=0$ otherwise. For the source relay-link, the source nodes decide on the subchannel allocation as noncooperative players. Assume first that each source node may choose at most one subchannel $p^{\prime}$ from the set of available subchannels. Let $\tau_{2}^{i}\left[p^{\prime}\right]=1$ if $i$ gets subchannel $p^{\prime}$ and let $\tau_{2}^{i}\left[p^{\prime}\right]=0$ otherwise. Assuming $i$ th source obtains assignment $\left(p, p^{\prime}\right)$, the utility of source nodes $i=1, \ldots, m$ can be defined as

$$
\gamma_{3}^{i}\left[p, p^{\prime}\right] \tau_{1}^{i}[p] \tau_{2}^{i}\left[p^{\prime}\right]=\frac{\gamma_{12}^{i}\left[p^{\prime}\right] \gamma_{23}[p]}{\gamma_{12}^{i}\left[p^{\prime}\right]+\gamma_{23}[p]+1} \tau_{1}^{i}[p] \tau_{2}^{i}\left[p^{\prime}\right]
$$

As $\gamma_{12}^{i}\left[p^{\prime}\right]$ depends of the source index $i$ and each source-torelay channel is independent of each other, the source nodes have different valuations for a given allocation $\left(p, p^{\prime}\right)$.

For a distributed allocation, a noncooperative game can be defined by the $m$ payoff functions (9); in a distributed noncooperative system each source maximizes its individual utility function taking as given the strategy choices of other source nodes.

In general each node may choose more than one subchannel for each link. Let $\mathcal{I}$ denote the set of subchannels available to all source nodes and let $\mathcal{J}$ denote the set of subchannels at relay. As an example, the utility of source $i$ could be defined as the sum

$$
u^{i}\left(\tau_{1}^{i}, \tau_{2}^{i}\right)=\sum_{p \in \mathcal{I}} \sum_{p^{\prime} \in \mathcal{J}} \gamma_{3}^{i}\left[p, p^{\prime}\right] \tau_{1}^{i}[p] \tau_{2}^{i}\left[p^{\prime}\right],
$$

where $\tau_{1}^{i}=\left\{\tau_{1}^{i}[p]\right\}$ and $\tau_{2}^{i}=\left\{\tau_{2}^{i}\left[p^{\prime}\right]\right\}$.

Fairness in a distributed channel-allocation game could be achieved via absolute resource constraints, e.g. requiring for each $i=1, . ., m$ :

$$
M \leq \sum_{p^{\prime} \in \mathcal{J}} \tau_{2}^{i}\left[p^{\prime}\right] \leq H,
$$

where $M$ denotes the minimum number of subchannel pairs to be assigned to a given source and $H$ denotes a maximum number of subchannel pairs to be assigned. At the relay, fairness can be formalized by analogous resource constraints:

$$
M \leq \sum_{p \in \mathcal{I}} \tau_{1}^{i}[p] \leq H
$$

DeFinition 3.1. A distributed subchannel allocation game can be defined by $m+1$ problems where each source $i=$ $1, \ldots, m$ maximizes the expected value of utility $u^{i}$ given in equation (10) with respect to $\tau_{2}^{i}$ subject to ith constraint (11) taking as given the allocation at the relay $\tau_{1}=\left\{\tau_{1}^{i}\right\}$; the relay node is the $m+1$ st player solving

$$
\max _{\left\{\tau_{1}^{i}\right\}} \sum_{i} u^{i}\left(\tau_{1}^{i}, \tau_{2}^{i}\right)
$$

Consider source node $i$ deciding on its subchannel allocation. In the absence of knowledge of the subchannel allocation at the relay, each source node expects the value of each subchannel allocation $p \in \mathcal{I}$ at relay to be equal to $E\left(\gamma_{23}[p]\right)>0$. From (6) is can be seen that, assuming $E\left(\gamma_{23}[p]\right)>0$, the preference ordering of subchannels $\left\{p^{\prime}\right\} \in \mathcal{J}$ implied by $\gamma_{3}^{i}\left[p, p^{\prime}\right]$ is the same as that implied by $\gamma_{12}^{i}\left[p^{\prime}\right]$. To simplify the distributed allocation problem, a simplified utility of source $i$ can be defined as:

$$
u_{s}^{i}\left(\tau_{2}^{i}\right)=\sum_{p^{\prime}} \gamma_{12}^{i}\left[p^{\prime}\right] \tau_{2}^{i}\left[p^{\prime}\right]
$$

where $\tau_{2}^{i}=\left\{\tau_{2}^{i}\left[p^{\prime}\right]\right\}$.

DeFinition 3.2. A simplified subchannel allocation game can be defined by $m+1$ problems where each source $i=$ $1, \ldots, m$ maximizes simplified utility $u_{s}^{i}\left(\tau_{2}^{i}\right)$, given in equation (14), subject to ith constraint (11) and the relay node solves problem (13) with utility $u_{i}$ in (10).

To define an equilibrium allocation, the concept of a noncooperative Nash equilibrium can be applied [3]. Letting $\tau_{2}^{-i}$ and $\tau_{1}^{-i}$ denote the subchannel allocations of users other than $i$ at the source and relay, respectively, Nash equilibrium $\tau_{2}^{i}, i=1, \ldots, m$ is defined by inequalities:

$$
u_{s}^{i}\left(\tau_{2}^{i}, \tau_{2}^{-i}\right) \geq u_{s}^{i}\left(\tau_{2}^{i \prime}, \tau_{2}^{-i}\right), i=1, \ldots, m,
$$

where $\tau_{2}^{i \prime} \neq \tau_{2}^{-i}, i=1, \ldots, m$.

Recent work in $[6,1]$ has specified conditions under which a Nash equilibrium can be found using a simple greedy iteration where each user chooses its individually optimal allocation given the strategy choices of other nodes.

Definition 3.3. Assume the source nodes update their subchannel strategies asynchronously in a random order. A distributed subchannel allocation algorithm can be defined for th iteration as the problem of source $i$ :

$$
\max _{\tau_{2}^{i}(t)} u_{s}^{i}\left(\tau_{1}, \tau_{2}^{i}(t)\right)
$$

subject to ith constraint (11).

Assume for simplicity that $\tau_{2}^{i}\left[p^{\prime}\right]$ and $\tau_{2}^{j}\left[p^{\prime}\right]$ (for subchannel $p^{\prime}$ ) are continuous for nodes $i$ and $j \neq i$. Then, in a congestion game (see e.g. [6]) the utility function $u^{i}$ of any player has the property:

$$
\frac{\partial u^{i}}{\partial \tau_{2}^{j}\left[p^{\prime}\right] \partial \tau_{1}^{i}\left[p^{\prime}\right]} \leq 0
$$

The game defined by payoff functions (10) or (14) (and constraints (11)) is a congestion game, cf. (7)-(8). With discrete $\tau_{2}^{i}\left[p^{\prime}\right] \in\{0,1\}, i=1, \ldots, m$, as it is assumed that a reserved link gives zero utility to any remaining player, the demand for a reserved subchannel is zero. A congestion game is a simple potential game with convergent greedy dynamics according to (16) [6].

A distributed solution to subchannel allocation need not be optimal from the point of view of a system-level objective. For a point of comparison, it is useful to consider the centralized allocation problem.

Definition 3.4. A centralized subchannel allocation problem can be defined as

$$
\max _{\left\{\tau_{1}^{i}, \tau_{2}^{i}\right\}} \sum_{i} u^{i}\left(\tau_{1}^{i}, \tau_{2}^{i}\right)
$$

subject to $m$ constraints (11).

The centralized problem, as posed above ${ }^{1}$, can be stated as a transportation problem [2] (a generalization of the assignment problem). The size of the cost matrix in the transportation problem is $m \times P \times P$ where $P$ denotes the number

\footnotetext{
${ }^{1}$ Alternatively, in a max-min fair centralized allocation, (18) can be replaced by an objective to maximize the minimum $u^{i}$.
} 
of source-relay subcarriers equaling the number of subcarriers at relay. The size of the transportation problem motivates the study of a distributed allocation scheme.

Recall that the relay node solves problem (13). Thus, the subchannel allocation problem at the relay node corresponds to the special case of the centralized allocation problem where the choices of the noncooperative source nodes $\left\{\tau_{2}^{i}\right\}$ are taken as given the outcome of the distributed allocation scheme (16). If each source may obtain at most one subchannel for the relay input and relay output, the solution to the allocation problem at the relay node can be obtained by solving an assignment problem ${ }^{2}$; the optimal solution corresponds to sorting the relay input and relay output channels according the selected metric (signal-to-noise ratio) and pairing ordered subchannels with each other ${ }^{3}$.

Following [7], subchannel allocation in the special case of a single source node is summarized in Appendix. For a single source an optimal assignment $\left(p, p^{\prime}\right)$ can be found by solving the assignment problem [2]. For a computationally efficient method for solving the assignment problem using majorization theory [10], see Appendix.

In general, different users may have different valuations of the quality of service measure $u^{i}$ or $u_{s}^{i}$; formally, the utility of user $i$ is some function $g_{i}\left(u_{i}\right)$ or $g_{i}\left(u_{s}^{i}\right)$ where $g_{i}$ can be different for different users. Congestion based pricing is a means for enforcing service differentiation [6]. A distributed subchannel allocation game with a resource price can be defined by payoff functions:

$$
g^{i}\left(u^{i}\right)-P_{s} \sum_{p^{\prime}} \tau_{2}^{i}\left[p^{\prime}\right], i=1, \ldots, m
$$

where $P_{s}$ denotes a unit price per subchannel allocation. The mechanism design problem is to define a price $P_{s}$ that guides the distributed system to an efficient resource allocation, corresponding to the solution of a centralized formulation with utilities $g^{i}\left(u^{i}\right)$.

\section{POWER ALLOCATION}

Thus far, transmit power control/allocation has been ignored, for simplicity assuming constant transmit power. In what follows, the subchannel allocation models presented above are extended to joint subchannel and power allocation.

With transmit power control, the link-specific signal-tonoise-ratio in the $p^{\prime}$ th subcarrier for the $i$ th source-relay link, and for the $p$ th subcarrier of the $i$ th relay-destination link, are defined as

$$
\gamma_{12}^{i}\left[p^{\prime}, r^{i}\left[p^{\prime}\right]\right]=\left|d_{12}\left[p^{\prime}\right]\right|^{2} r^{i}\left[p^{\prime}\right] / \sigma_{2}\left[p^{\prime}\right]^{2},
$$

and

$$
\gamma_{23}^{i}\left[p, r^{i}[p]\right]=\left|d_{23}[p]\right|^{2} r^{i}[p] / \sigma_{3}[p]^{2},
$$

where $r^{i}\left[p^{\prime}\right]$ denotes the power allocated at source node $i$ to the $p^{\prime}$ th subcarrier and $r^{i}[p]$ denotes the power allocated to subcarrier $p$ of node $i$ at the relay. Using the above nota-

\footnotetext{
${ }^{2}$ Assignment problem and its solution are summarized in Appendix, assuming a single source node.

${ }^{3}$ Assuming at most a single subchannel per user, the solution to the channel allocation problem at the relay is analogous to that presented in Appendix.
}

tions, $\gamma_{3}^{i}\left[p, p^{\prime}\right]$ can be generalized, for $i=1, \ldots, m$, to

$$
\gamma_{3}^{i}\left[p, p^{\prime}, r^{i}\left[p^{\prime}\right], r^{i}[p]\right]=\frac{\gamma_{12}^{i}\left[p^{\prime}, r^{i}\left[p^{\prime}\right]\right] \gamma_{23}^{i}\left[p, r^{i}[p]\right]}{\gamma_{12}^{i}\left[p^{\prime}, r^{i}\left[p^{\prime}\right]\right]+\gamma_{23}^{i}\left[p, r^{i}[p]\right]+1} .
$$

With transmit power control, the utility of source $i$ could be defined as the sum

$$
u^{i}=\sum_{p \in \mathcal{I}_{i}} \sum_{p^{\prime} \in \mathcal{J}_{i}} \gamma_{3}^{i}\left[p, p^{\prime}, r^{i}\left[p^{\prime}\right], r^{i}[p]\right]
$$

where $\tau_{1}^{i}, \tau_{2}^{i},\left\{r^{i}[p]\right\},\left\{r^{i}\left[p^{\prime}\right]\right\}$ are the arguments of $u_{i}, i=$ $1, \ldots, m$. Transmit power constraint for source node $i$ can be stated as

$$
\sum_{p^{\prime}} r^{i}\left[p^{\prime}\right] \leq R_{2}^{i}
$$

where $R_{1}^{i}$ is the power constraints of source node $i$. Analogously, the $i$ th power constraint at relay node can be stated as:

$$
\sum_{p} r^{i}[p] \leq R_{1}^{i}, i=1, \ldots, m
$$

where $R_{2}^{i}$ is the power constraints of $i$ at relay.

DEFINITION 4.1. The resource allocation game for joint subchannel and power allocation can be defined by $m$ utility functions (21) to be maximized subject to source-specific constraints (11) and (22).

The relay node can be assumed to simultaneously solve the power allocation to different users and subchannels, taking as given the noncooperative power allocations for the sourcerelay link.

The resource allocation game with respect to simultaneous subchannel selection and transmit power allocation also is a congestion game; formally, due to (7)-(8):

$$
\frac{\partial u^{i}}{\partial r^{j}\left[p^{\prime}\right] \partial r^{i}\left[p^{\prime}\right]} \leq 0, \forall j \neq i, \forall p^{\prime} \in \mathcal{I}
$$

A centralized subchannel and power allocation problem corresponding to simultaneously solving the game problem in Definition 4.1 can be stated as the problem:

$$
\max \sum_{i=1}^{m} u^{i}\left(\tau_{1}^{i}, \tau_{2}^{i}, r^{i}\left[p^{\prime}\right], r^{i}[p]\right)
$$

subject to $m$ sets of constraints (11), (22) and (23).

\section{PERFORMANCE}

The models for subchannel allocation with and without power control presented above can be numerically evaluated by extending the simulation model in [7] to multiple source nodes and power control. For simplicity, we consider the simplified game according to Definition 3.2.

We assume that each source-to-relay and the relay-todestination channel have each a Rayleigh distribution with $L$ iid multipath components. The average $S N R$ of each subcarrier is set to $6 \mathrm{~dB}$ in each link and there are $P=64$ subcarriers available. With sixteen users, each user is allocated 16 subcarriers in both source-to-relay and relay-todestination link. In the experiment with four users, each user has sixteen subcarriers. Thus, all subcarriers are always used. The relay and source nodes in the considered 
two-hop scheme are orthogonal, e.g., they are assumed to be transmitting during different slots.

The users in the source-to-relay link select the subchannel indices sequentially from the set of remaining subchannels. This set is known to each source via vector $\mathbf{z}_{1}$, which is broadcasted from the relay node to each source node after a given user has reserved her subchannels at the relay node. The first user is in a preferred position, in that she selects the indices of $P_{1}$ best subchannels from $P$ available subchannels. The next source selects the best subchannels from $P-P_{1}$ subchannels, and so on. The last user in the selection process is left with $P_{m}$ subchannels, i.e. those that other sources did not appreciate. Hence, first source obtains largest selection diversity gain, and the last has no selection diversity gain. As explained above, with four users $P_{i}=16, \forall i$ and with sixteen users $P_{i}=4, \forall i$, and in both cases $\sum_{i} P_{i}=P$.

The selection diversity effect is depicted most clearly with the solid lines in Fig. 2. Therein (labeled with "No Assignment"), the relay keeps the same the same subchannel order when transmitting. Optimal assignment refers to the optimal assignment of output subchannels at relay. this is obtained as the solution to problem (13), taking as given distributed source-to-relay subchannel allocation. The first user (the one that first makes the selection) is able to increase the capacity significantly as multipath spread increases. In contrast, user sixteen is allocated the remaining four subchannels, and the capacity is unaffected by the multipath spread of the channel.

The fairness aspect of opportunistic channel allocation and in particular the effect of the optimal assignment is seen by comparing the solid and dashed lines in Fig. 2. It is seen that the optimal assignment further increases the disparity of the first and last users in the selection process. This is due to Proposition 6.1 in Appendix. Fig. 3 shows the spectral efficiency of the system with different number of source nodes. It is seen that the total capacity (spectral efficiency) increases when the number of source nodes increases. This is a combined effect of selection diversity and optimal channel assignment at relay.

The Figures are drawn as a function of multipath spread, since this parameter, together with the number of users, affects the channel variability. Increasing either the number of users or multipath spread increases the performance of the opportunistic system. For example, in a flat fading channel (with $L=1$ ) each source-to-link is obviously indifferent to the actual subchannel indices as all subchannels are identical. When $L$ increases the selected subchannel indices are scattered or interlaced across the $P$ available frequency bins. For example, considering the first source, in a frequencyselective multipath channel the powers of the best $P_{1}<P$ subchannels are clearly above the average. Similarly for the others, apart from the last source. This explains the fact that the average performance improves as $L$ increases. With optimal assignment, the last user suffers for the benefit of the total capacity.

In above examples, optimal assignment refers to optimal assignment at the relay node. The corresponding subchannel allocation need not be globally optimal; the comparison of the performance of distributed subchannel allocation to centralized allocation according to Definition 3.4 is left for future work.

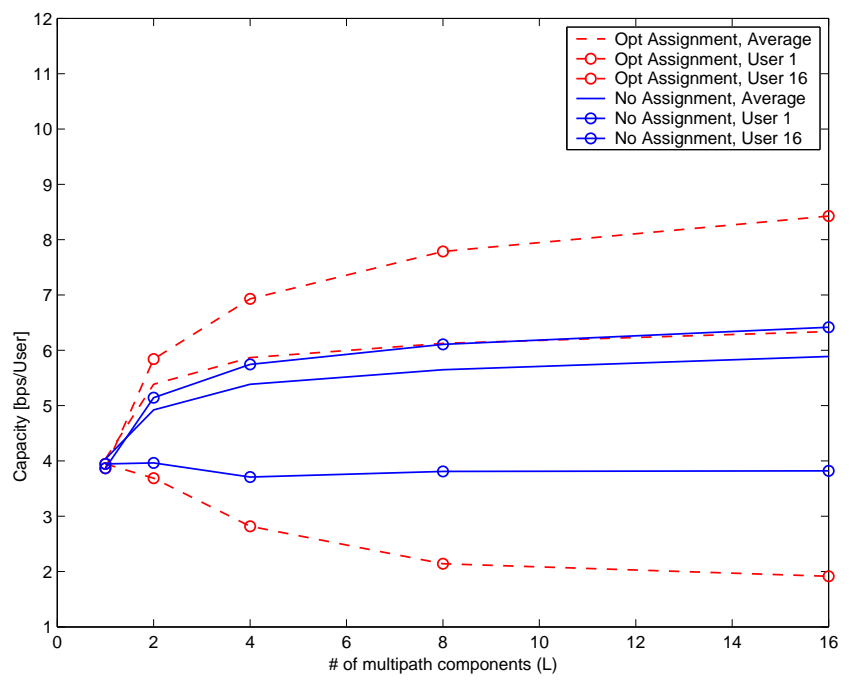

Figure 2: Capacity at destination node for first, last and the average user in a multipath channel.

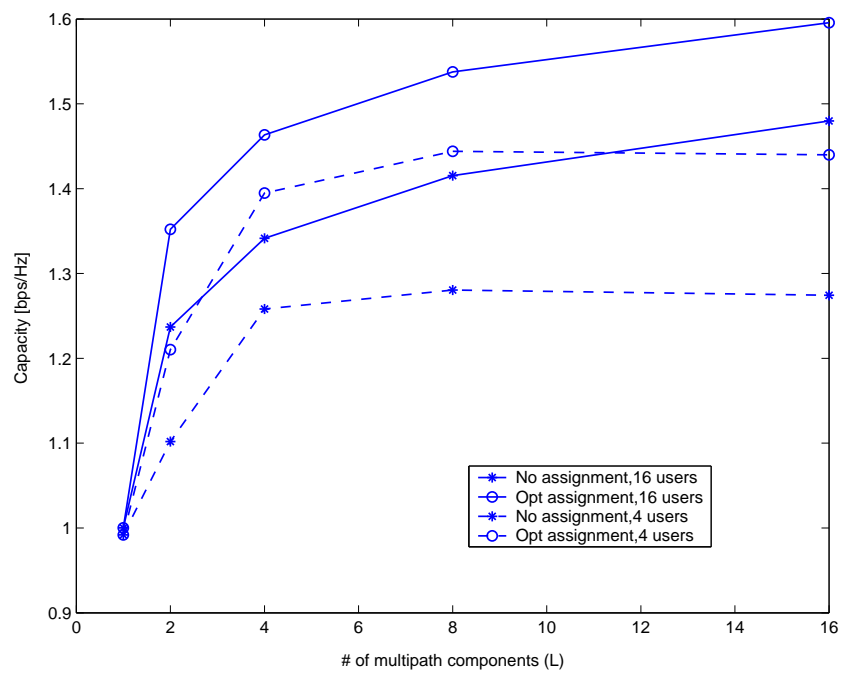

Figure 3: Comparison of the average capacity at destination in a multipath channel with four and sixteen users. 


\section{CONCLUSION}

The subchannel assignment problem for amplify and forward relays is formulated as a distributed resource allocation problem. Each user is modeled as a noncooperative player in a resource allocation game. Distributed subchannel assignment algorithms are proposed for relay systems. Numerical examples assuming constant transmit power illustrate the performance of distributed subchannel assignment; opportunistic channel allocation implies a trade-off between the average capacity and fairness.

Performance evaluation of subchannel assignment with power control is left for future work. Another topic for future work is to consider multiple relay nodes in a distributed multi-hop network.

\section{ACKNOWLEDGEMENTS}

The first author is supported by the STREP project No. IST-026905 (MASCOT) within the sixth framework programme of the European Commission

\section{REFERENCES}

[1] E. Altman and Z. Altman, S-modular games and power control in wireless networks, IEEE Tr. Automatic Control, Vol. 48, No. 5 May 2003, pp. 839-842

[2] M. Bazaraa, J. Jarvis and H. Sherali, Linear Programming and Network Flows, Wiley, 1990

[3] D. Fudenberg and J. Tirole, Game Theory, The MIT Press, 1993

[4] J. Gross, S. Valentin, H. Karl, and A. Wolisz, A study of impact of inband signaling and realistic channel knowledge for an example dynamic OFDM-FDMA system, European Transactions on Telecommunications, Vol. 16, pp. 37-49, 2005.

[5] I. Hammerström and A. Wittneben, Joint power allocation for nonregenerative MIMO-OFDM relay links, in Proc. International Conference on Acoustics, Speech, and Signal Processing (ICASSP), Toulouse, France, May 2006.

[6] T. Heikkinen, A potential game approach to distributed power control and scheduling, Computer Networks 50(13), 2006, pp. 2295-2311

[7] A. Hottinen and T. Heikkinen, Subchannel assignment in OFDM relay nodes, in Proc. CISS 2006, Princeton, NJ, USA, March 22-24 2006.

[8] A. Hottinen and T. Heikkinen, Optimal subchannel assignment in a two-hop OFDM relay, in Proc. IEEE Workshop on Signal Processing Advances in Wireless Communications, Helsinki, Finland, June 17-20 2007.

[9] D. E. Knuth, The Art of Computer Programming, Volume 3: Sorting and Searching. 2nd Edition, Reading, Massachusetts, Addison-Wesley, 1998

[10] A. W. Marshall and I. Olkin, Inequalities: theory of majorization and its applications, Academic Press, New York, 1979

[11] G. Stüber, J. B. Barry, S. W. McLaughlin, Y. Li, M.A. Ingram and T. G. Pratt, Broadband MIMO-OFDM wireless communications, Proc. IEEE, Vol. 92, No. 2, Ferbuary 2004, pp. 271-294

[12] L. Wolsey, Integer Programming, 1998, Wiley

[13] http://www.ieee802.org/16/relay/index.html

\section{APPENDIX}

\section{Subchannel Assignment with Single Source}

Following $[7,8]$, define a performance indicator $\phi_{p, p^{\prime}}$ for a given subchannel $\left(p, p^{\prime}\right)$ pair. The performance indicators are captured in matrix $\boldsymbol{\Phi}=\left[\phi_{p, p^{\prime}}\right]$. Variable $t_{p, p^{\prime}}=1$ if pair $\left(p, p^{\prime}\right)$ is assigned and $t_{p, p^{\prime}}=0$ otherwise. The assignment problem then is given as

$$
\max \sum_{p} \sum_{p^{\prime}} \phi_{p, p^{\prime}} t_{p, p^{\prime}}
$$

subject to

$$
\begin{gathered}
\sum_{p} t_{p, p^{\prime}}=1, \forall p^{\prime} \\
\sum_{p^{\prime}} t_{p, p^{\prime}}=1, \forall p, \\
t_{p, p^{\prime}} \geq 0, \forall p, p^{\prime}
\end{gathered}
$$

The optimal solution is known to be integral, with $t_{p, p^{\prime}} \in$ $\{0,1\}$. Due to constraints $(25)-(26)$ the resulting $\mathbf{T}=\left[t_{p, p^{\prime}}\right]$ is a permutation matrix. The complexity of the classical primal-dual assignment algorithm for problem (24)-(27) is $O\left(n^{4}\right)[12]$.

To reduce complexity, rewrite (6) in functional form

$$
\phi\left(\gamma_{12}, \gamma_{23}\right)=\left(\gamma_{12} \gamma_{23}\right) /\left(\gamma_{12}+\gamma_{23}+1\right) .
$$

Since the optimal solution is known to be a permutation matrix, the subchannel assignment problem is equivalent to

$$
\mathbf{T}^{*}=\arg \max _{\mathbf{T}=\left[t_{p, p^{\prime}}\right]} \sum_{p=1}^{P} \sum_{p^{\prime}=1}^{P} t_{p, p^{\prime}} \phi\left(\gamma_{12}\left[p^{\prime}\right], \gamma_{23}[p]\right),
$$

where $\mathbf{T}$ is a permutation matrix. The solution to (28) can be found efficiently via simple sorting-based algorithms, provided that certain conditions are satisfied.

Proposition .1. Let $\gamma_{12}\left[p_{l}^{\prime}\right]$ denote the lth largest element of $\left\{\gamma_{12}\left[p^{\prime}\right]\right\}_{p^{\prime}=1}^{P}$ and let $\gamma_{23}\left[p_{l}\right]$ denote the lth largest element of $\left\{\gamma_{23}[p]\right\}_{p=1}^{P}$. The optimal solution to (28) with $L$-superadditive performance indicator function $\phi$ is one in which source-relay subchannel $p_{l}^{\prime}$ is paired with relay-destination subchannel $p_{l}$.

Proof: Omitted for brevity (see [8]).

Recall from [10] that a real-valued twice differentiable function $\phi$ defined on $\mathbb{R}^{2}$ is $L$-superadditive if

$$
\frac{\partial^{2}}{\partial s \partial r} \phi(r, s) \geq 0 \text {. }
$$

Using condition (29), it is easy to verify that functions

$$
\phi_{1}\left(\gamma_{12}, \gamma_{23}\right)=\left(\gamma_{12} \gamma_{23}\right) /\left(\gamma_{12}+\gamma_{23}+1\right)
$$

and

$$
\phi_{2}\left(\gamma_{12}, \gamma_{23}\right)=\log _{2}\left(1+\left(\gamma_{12} \gamma_{23}\right) /\left(\gamma_{12}+\gamma_{23}+1\right)\right)
$$

are $L$-superadditive on non-negative orthant. Thus, the optimal assignment that is obtained by sorting $\gamma_{\mathbf{1 2}}$ and $\gamma_{23}$ is identical for both indicators. Interestingly, $L$-superadditivity plays also a role in distributed power allocation $[1,6]$.

The computational complexity of various sorting algorithms are well-known, and typically amount to $O(n \log (n))$, see e.g. [9]. The complexity of the classical primal-dual assignment algorithm is $O\left(n^{4}\right)$ [12]. 